
\title{
28 Research Square \\ The Expression of MMP-2 on the Pressure Side During Tooth Movement
}

\section{Jing Zhang}

Feixian People's Hospital

\section{Qiuqiu Chen}

University of traditional Chinese Medicine

\section{Fuxiang Luo}

Xinjiang Uygur Autonomous Region

Hong Liu ( $\nabla$ hongliuortho@163.com )

Affiliated Hospital of Traditional Chinese Medicine, Xinjiang Medical University

\section{Research Article}

Keywords: MMP-2, Orthodontic, Tooth movement, Root resorption, Immunohistochemistry

Posted Date: October 19th, 2021

DOl: https://doi.org/10.21203/rs.3.rs-953737/v1

License: (9) This work is licensed under a Creative Commons Attribution 4.0 International License. Read Full License 


\section{Abstract}

Objectives: To explore the effects of matrix metalloproteinase-2 (MMP-2) on the development of orthodontic root resorption in rats and to provide a theoretical basis for prevention and treatment of root resorption in clinical diagnosis.

Methods: Thirty-five 6-8-week-old male SD rats were randomly divided into 7 groups, each with 5 rats weighing approximately $200 \mathrm{~g} \pm 20 \mathrm{~g}$. An orthodontic traction device was applied between the right first molar and the maxillary incisor in the experimental rats, and a mesial tractive force of $60 \mathrm{~g}$ was applied. Five rats were used for the control group without orthodontic applications. After 0, 1, 3, 5, 7, 14, and 21 days, the maxillae were abducted, and immunohistochemical analysis was performed. We used hematoxylin eosin staining to observe root resorption. The expression of MMP-2 was also assessed by immunohistochemical staining. SPSS17.0 statistical software was used to process the data.

Results: Forcing $3 \mathrm{~d}$ was observed in the formation of bone resorption lacunae, which were confined to the cementum. After that, it becomes more serious. The most serious damage in 7 days. In $14 \mathrm{~d}$, root absorption was relieved, and osteogenic cells aggregated at the root absorption site. Root resorption lacuna visible dentin repair phenomenon. With the extension of the force application time, the expression of MMP-2 was raised gradually, reached the peak at $7 d$, and then gradually declined, $21 d$ tends to be stable, but still stronger than controls.

Conclusion: MMP-2 is involved in the metabolism of extracellular matrix in the process of root absorption.

\section{Introduction}

Root resorption is an inevitable side effect during orthodontic tooth movement and can be expected to result in a compromise in the prognosis of the tooth and the stability of the treatment results[1, 2]. It arises in orthodontic load-induced sterile inflammation that results in resorption of the superficial root cementum. It can also become more severe with eventual resorption of the underlying dentin[3]. Feller et al indicated that cellular cementum can repair apical external root resorption but nevertheless may bring about permanent loss of root length[4]. Some histological studies have reported that OIRR occurs in approximately $90 \%$ of orthodontic teeth during movement $[5,6]$, and some studies have shown that $1-5 \%$ of orthodontically treated teeth develop severe root resorption exceeding $4 \mathrm{~mm}[6]$.

The movement of orthodontic teeth involves complex biological processes characterized by the response of periodontal tissue to biomechanical forces. Root absorption may involve many factors, especially duration, magnitude, and types of orthodontic force applied, which are considered to be factors affecting root resorption [7]. Previous investigations have shown that proinflammatory cytokines may affect the pathogenesis of external root resorption during tooth movement[8-10]. 
Matrix metalloproteinases (MMPs) are zinc-dependent matrix proteins belonging to the metalloproteinase M10A subfamily and have long been recognized as the major effector of extracellular matrix (ECM)/core matrix protein degradation [11]. Matrix metalloproteinases (MMPs) are the main enzymes involved in ECM degradation. Under normal conditions, they become less active but are activated during repair or remodeling, as well as in diseased or inflamed tissues [12]. Cantarella et al exerts force on the left teeth of the upper jaw, extracts MMP-1 and MMP-2 and detects their content in gum trench fluid and finds that the pressure side and tension side of the affected teeth and the content of MMP-I and MMP-2 in the gum trench fluid increase in the early stage of the force[13]. They inferred that MMP-2 plays an important role in the early stages of orthodontic treatment.

Therefore, based on increasing evidence, we speculate that MMP-2 might be involved in orthodontic root resorption. However, there is little literature on the relationship between matrix metalloproteinases and orthodontic root resorption. Hence, the study aimed to evaluate the relationship between orthodontic root resorption during tooth movement and the expression of MMP-2 by establishing a disability root absorption animal model of SD rat orthodontic teeth and immunohistochemistry staining. We are expected to provide new ideas for the clinical prevention and treatment of this unwelcome event.

\section{Materials And Methods}

All experimental procedures in this study were reviewed and approved by the Ethics Committee of Xin Jiang Medical University and were in accordance with the "Guiding Opinions on Treating Experimental Animals" issued by the Ministry of Science and Technology in 2006. This study also conforms to the ARRIVE guidelines.

\section{Animals:}

Thirty-five Sprague-Dawley rats were purchased from the Animal Experiment Center of Xinjiang Institute of Traditional Chinese Medicine. Eight-week-old SD

rats(weight, $200 \pm 20 \mathrm{~g}$ ) were used in this study. Rates provided by the Experimental Animal Center of Xinjiang Uygur Institute of Traditional Chinese Medicine were allowed 1 week to acclimatize to their new laboratory environment. During the experiment, all animals were individually placed in a plastic cage at the Medical Experimental Research Center and fed standard pellets and water ad libitum. The rats were randomly divided into one control (without tooth movement force) and six experimental tooth movement groups (five animals in each group). The health status of each mouse was assessed by daily weight monitoring. Local ethical committee approval was gained.

\section{Application of Orthodontic Devices and Retention}

Except for the control group without any treatment (directly sacrificed and taken), another experimental group of rats was anesthetized by intraperitoneal injection of ketamine hydrochloride $(100 \mathrm{mg} / \mathrm{kg})$ and fixed on a mouse plate, and the abdomen was placed upward. The application of orthodontic 
inflammatory root resorption was a modified version of that previously described[14]. In addition, using a slow-speed dental handpiece(SAESHIN PRECISION INDICO) to grind a shallow groove of approximately $0.5 \mathrm{~mm}$ deep in the right middle jaw of the right maxillary first molar and the cervical third of the incisors of each rat; The orthodontic nickel-titanium tension spring (wire size, $0.2 \mathrm{~mm}$; diameter, $1 \mathrm{~mm}$; Smart Technology, Beijing, China) was ligated between the maxillary first molar and the maxillary central incisor of each rat, and the upper anterior teeth were anchorage, with $0.6 \mathrm{~N}$ Force value pulling the maxillary first molar to move mesial (Fig. 1.A). The magnitude of orthodontic force was measured by a tension gauge. The experimental tooth movement lasted for 1, 3, 5, 7, 14 and 21 days.

\section{Preparation of materials and tissue sections:}

The mice were sacrifificed after 0, 1day, 3day,5day,7day,14day,21day(Fig. 1.B). The maxillary right first molar and its surrounding alveolar bone were completely removed and immediately fixed in $40 \mathrm{~g} / \mathrm{L}$ polyformaldehyde solution for 24 hours and then decalcified in EDTA solution at $0.5 \mathrm{~mol} / \mathrm{L}$. The decalcified specimens were dehydrated by gradient ethanol and embedded in paraffin. The specimens were sliced in the sagittal direction along the long axis of the teeth, and the thickness of the specimens was approximately $5 \mu \mathrm{m}$.

\section{Hematoxylin-Eosin Staining of Maxillary Parasagittal Sections:}

The steps of HE staining were as follows: 1) paraffin sections were dewaxed and dehydrated; 2) incubated in 0.2MTris hydrochloric acid buffer (PBS, Beijing Zhongshan Bioengineering) and incubated at $37^{\circ} \mathrm{C}$ for 1 hour; 3 ) rinsed with double steamed water 3 times and dried at room temperature after PBS rinsing; 4) the slices were fully immersed in TRAP staining solution and incubated at $37^{\circ} \mathrm{C}$ for $50 \mathrm{~min} ; 5$ ) distilled water was washed and dried at room temperature; 6) hematoxylin restained; 7) neutral gum seal; 8) under microscope, the periodontal tissue and alveolar bone resorption of the pressure side and tension side of maxillary first molar were observed.

\section{Immunohistochemical Staining:}

After paraffin section dewaxing and hydration, APES was selected for slide decalcification, and the slice was carefully adhered to. It was placed in a $58^{\circ}$ oven for 45 minutes and placed at room temperature for 10 minutes. The purpose of dewaxing and hydration was to destroy the activity of endogenous enzymes and rinse them with distilled water 3 times. The slices were fully immersed in $0.01 \mathrm{~m}$ PBS buffer $(\mathrm{pH}=6.0)$ by the thermal repair antigen method. The microwave oven was heated to boiling and stopped heating at intervals of $5 \mathrm{~min}$ and $10 \mathrm{~min}$, repeated once and twice. PBS washing after cooling (PH7.2-7.6) Wash 1 times twice. Add 5\%BSA sealing solution, room temperature $30 \mathrm{~min}$, dump more than liquid, do not rinse. Drip $1 \leq 100$ diluted MMP-2 antibody, $4{ }^{\circ}$ overnight, PBS instead of first antibody as negative control, PBS (pH 7.2-7.6) 2 minutes interval washing 3 times. Anti-goat anti-mouse IgG, $37^{\circ} 20 \mathrm{~min}$. PBS washed $5 \mathrm{~min}$ 4 times. A DAB color kit was used, $1 \mathrm{ml}$ distilled water was added, and reagents $A, B$, and $C$ were mixed well. Add it to the slice. Color at room temperature, control reaction time under mirror, wash with distilled 
water 5-30 min. Hematoxylin restaining. Dehydrated, transparent, neutral gum seal. Microscopic observation.

\section{Statistical analysis:}

The positive expression of MMP-2 was present as brown and yellow granules, and the results of immunohistochemistry and semidefinite analysis were performed. Using an optical microscope(Olympus. Japan), a photography system and Image-Pro Pro Plus 6.0 professional image analysis software, and semiquantitative analysis of each slice was carried out. The proximal middle side (compression Wanfang data 10 force side) and far middle side (stretch side) of the experimental molars were selected as the measuring sites. At the same light intensity, three high magnification horizons per slice were randomly selected to calculate the target area, cumulative optical density and average optical density. The final results were represented by the average optical density and statistically analyzed.

After using Image-Pro Pro Plus 6.0 specialized software to obtain the average optical density of the pressure region with different loading times, the data were measured. The results are statistically processed by SPSS 17.0. The ratio of the photo group to the actual group showed a positive state distribution and variance homogeneity, and single factor variance analysis (one-way ANOVA) was used.

\section{Results}

Control group: The collagen fibers were arranged evenly and regularly, and the width of the periodontal ligament was approximately the same. Fibroblasts were uniformly distributed among the main fiber bundles, which was consistent with the long axis of the principal fiber bundles. The edge of alveolar bone was neat, and no osteoclasts were found (Fig. 2.区).

Experimental groups: When the force was applied for 1 day, the gap of the distraction-side periodontal membrane was widened, and the periodontal membrane gap of the compression-side side was narrowed. A small number of osteoclasts were found in the periodontal tissue and periapical area of the pressure side. In the $3 \mathrm{~d}$ (Fig. 2. \)and $5 \mathrm{~d}$ (Fig. 2. .)groups, osteoclast aggregation on the pressure side, obvious vitreous degeneration was observed (Fig. 3), and the formation of bone resorption lacunae (limited to cementum) and the irregular arrangement of fibroblasts near the lacunae were observed. The number of osteoclasts in the 5-day group(Fig. 2.『) was significantly higher than that in the 3-day(Fig. 2.『)group. In the 7-day group(Fig. 2.『), there was clear root resorption at the root tip and root bifurcation of the teeth and involved dentin. A large number of polynuclear osteoclasts were discovered in the resorption lacunae, latent bone resorption took place, and the infiltration of inflammatory cells was obvious. Osteoblast aggregation and osteoclast number decreased in the root resorption site of the 14-day group (Fig. 2.区), and the degree of root absorption and vitreous degeneration decreased significantly in the 21-d group (Fig. 2.『).

\section{Immunohistochemical:}


Compared with the control group(Fig. 3.A), the level of MMP-2 was found in the periodontal tissues of each experimental group by using Image-Pro Plus 6.0 professional image analysis software and data analysis software. The positive expression of MMP-2 is represented by brown yellow granules. The results showed that the expression of MMP-2 increased gradually on the 1st day, on the 3rd day(Fig. 3.B), increased gradually on the 5th day(Fig. 3.C), reached a peak on the 7th day(Fig. 3.D), decreased gradually on the 14th day(Fig. 3.E), and finally tended to be stable on the 21 st day(Fig. 3.F), but it was still slightly higher than that of the blank control group(Fig. 4).

The expression and localization of MMP-2 were mainly in periodontal tissue and were weakly expressed in dental pulp tissue. Compared with the control group, there was no significant difference in the pressure side of the pressure side between the two groups on the 1st and 14th day $(P>0.05)$, but there was no significant difference on the 21 st day between the first day and the 14th day $(P>0.05)$, and there was no significant difference on the 14th day between the 1st day and the 14th day $(P>0.05)$, and there was no significant difference on the 14th day between the 5th day and the 7th day $(P>0.05)$, and there was no significant difference on the 14th day between the 5 days and the 7 th day $(P>0.05)$, but there was no significant difference between the 5 days and the 7th days $(P>0.05)$, but there was no significant difference between the 5 days and 7 days $(P>0$. There was no significant difference on the 21 st day between the 14th day and the 7th day $(P>0.05)$, but the rest were statistically significant $(p<0.05)$. The pressure side of different loading time groups was analyzed by single factor variance analysis. The results showed that there was no significant difference between the control group and the experimental group on the 1st day, the 3rd day, the 14th day, the 21 st day, the 3rd day and the 3rd day, the 14th day, the 21 st day, the $3 \mathrm{D}$ and 5 th days, the 14 th day, the 14 th day, the 7 th day, the 14 th day, the 14 th day, the 21 st day, the 7th day and the 7th day, the 14th day, the 21 st day, the 14th day, the 14th day, the 21 st day, the 14th day and the 21st day(Table.1). There was a significant difference in statistics $(P>0.05)$, and there was a significant difference in the rest $(p<0.05)$.

\section{Discussion}

Orthodontic root absorption in rat molars is a useful model for investigating the mechanism of dental root resorption. In this research, we used SD models to investigate whether MMP-2 is involved in orthodontically induced inflammatory root resorption (OIIRR). We demonstrated the expression of MMP-2 in rat root resorption and the localization of this enzyme in osteoblasts, osteoclasts and fibroblasts. Based on immunohistochemical analysis, a high level of MMP-2 expression was revealed in the rat root resorbing tissue, indicating that MMP-2 should take part in root resorption during orthodontic tooth movement.

Root resorption is an unwelcome effect of orthodontic treatment. Although bone resorption craters have been shown to be cured by cementation, the apical area is separated from the rest of the root, and the root will be permanently shortened. The process of root resorption is closely related to injury and necrosis of the PDL. When a heavy orthodontic force is used for a sustained period of time (weeks or months), necrosis (transparency) of the compressed PDL may develop rapidly [15]. According to previous studies, 
the establishment of orthodontic teeth movement of Model in rats, $50 \mathrm{~g}$ or so of nickel-titanium helical pull force allows teeth to move effectively, along with force increasing, the speed at which the teeth move is reduced, and the probability of root absorption occurs increases, and can cause excessive damage to periodontal tissue[16]. As the force increases, the area where absorption occurs increases. To reduce the occurrence of root absorption, they advocate the use of light force in the clinic[17]. In a study of the movement of common rat teeth, the magnitude of the correction force set in the model was $20 \mathrm{~g}-50 \mathrm{~g}[18]$. Therefore, the force value of the nickel-titanium tension spring set in this experiment was $60 \mathrm{~g}$ to ensure that the orthodontic force-induced animal model could be successfully established. Histological appearance of root resorption in HE staining show that when the force 7d, the root tip of the tooth and the root fork are visible in the root absorption phenomenon, and tired of the essence of the tooth, absorption of a large number of multicore bone-breaking cells visible in the trap, and can be seen that the potential bone absorption is taking place, inflammatory cells immersion is obvious, indicating the successful establishment of orthodontarosic mobility root absorption model.

Brudvik and Rygh studied mice and rats and confirmed that OIIRR is part of the transparent strip elimination process[19-24]. As noted above, MMPs are the major enzymes involved in the degradation of ECM. Under normal conditions, their activity is low, but their activity is elevated during the repair or remodeling of diseased or inflammatory tissues [12]. Tissue inhibitors of metalloproteinases (TIMPs), a major endogenous inhibitor of metalloproteinases, reversibly inhibit MMPs in a 1:1 stoichiometry [25]. MMP-2 is collagens (IV, V, VII, X), gelatin, elastin. Our results showed that orthodontic forces affect both MMP-2 protein levels on the compression and tension sides, although to different extents, MMP-2 protein levels change in a time-dependent fashion (table 1). In the control group, the expression of MMP-2 was at baseline levels(Fig. 3.A), which was similar to that at 1 day. Then, the expression of MMP-2 was induced by compression and increased significantly in a time-dependent fashion, reaching a peak after 7 days of force application and then gradually returning to weakly positive at 21 days. Studies have demonstrated that before the decomposition of the matrix, there is an enzyme formation and storage phase, followed by protease decomposition and matrix destruction[26]. Therefore, we conclude that the $1 \mathrm{~d}$ and $3 \mathrm{~d}$ of the intensification in this experiment are the formation stages of the enzyme, so the content is slightly increased. After 5 days of exertion, obvious root resorption occurred, and the enzyme content increased significantly. After 7 days of intensification, the expressive content reached the maximum(Fig. 3.E). The disordered arrangement of periodontal fibers hinders the cushioning effect of the periodontal ligament on the orthodontic force, resulting in a relative increase in local pressure, which leads to an increase in the level of relevant inflammatory mediators, which accelerates the activation of osteoclasts and ultimately aggravates inflammatory root resorption. This is part of the possible reasons. Then, it gradually declines until $21 \mathrm{~d}$ after the force, and the content reaches a dynamic stable level, with no obvious fluctuations(Fig. 3.F).Previous investigations proved that decomposition of the matrix is the result of the interaction of TIMPs and MMPs[27, 28]. As the expression of MMPs increases, TIMPs also increase, inhibiting the excessive degradation of MMPs to tissues. Both MMPs and TIMPs are obtained by adult periodontal ligament fibroblasts. The degradation of periodontal ligament tissue may be related to the increase in local MMP and the decrease in TIMPs. This may be explained by the inhibition of MMP-2 
expression at the beginning of TIMPs, so the expression of MMP-2 is decreased in the late stage of posteenergization. In addition, it may be accompanied by the repair of the root tissue during the root resorption process, the root inflammation is relieved, and the released necrosis is reduced so that MMP-2 is not excessively produced. At 21 days, the expression of MMP-2 in periodontal tissues was significantly reduced(Fig. 3.F), but it was still more than the physiological state, suggesting that periodontal tissues are still actively remodeling and reconstructed until the internal environment state is stable.

The statistical results showed that there was a significant difference in expression levels between the control group and each experimental group, indicating that MMP-2 is sensitive to root resorption, and we conclude that it may be involved in root resorption.

In conclusion, the results confirmed the hypothesis that MMP-2 plays a significant role in OIIRR. However, it is unclear how MMP-2 works and how it can inhibit root resorption. This is also the focus of the next group.

\section{Declarations}

No potential conflicts of interest relevant to this article were reported.

\section{Author contributions}

Hong Liu contributed to the conception and design of the study;

Jing Zhang and Fuxiang Luo performed the experiments;

Jing Zhang and Hong Liu contributed significantly to the analysis and manuscript preparation;

Jing Zhang and Hong Liu performed the data analyses and wrote the manuscript;

Hong Liu and Qiuqiu Chen helped perform the analysis with constructive discussions.

All authors read and approved the final manuscript.

\section{References}

1. Liu, Z. et al. Ultrasound enhances the healing of orthodontically induced root resorption in rats. Angle Orthod, 82 (1), 48-55 (2012).

2. Seifi, M. et al. Analysis of Transforming Growth Factor- $\beta 1$ Expression in Resorptive Lacunae following Orthodontic Tooth Movement in An Animal Model[J]. Cell Journal, 19 (2), 278-282 (2017).

3. Sawicka, M. et al. Interrupted orthodontic force results in less root resorption than continuous force in human premolars as measured by microcomputed tomography[J]. Folia Histochemica et Cytobiologica, 52 (4), 289 (2014). 
4. Feller, L. et al. Apical External Root Resorption and Repair in Orthodontic Tooth Movement: Biological Events. Biomed Res Int, 2016. 2016: p. 4864195.

5. Elhaddaoui, R. et al. Resorption of maxillary incisors after orthodontic treatment-clinical study of risk factors. Int Orthod, 14 (1), 48-64 (2016).

6. Roscoe, M. G., Meira, J. B. \& Cattaneo, P. M. Association of orthodontic force system and root resorption: A systematic review. Am J Orthod Dentofacial Orthop, 147 (5), 610-626 (2015).

7. Topkara, A., Karaman, A. I. \& Kau, C. H. Apical root resorption caused by orthodontic forces: A brief review and a long-term observation[J]. European Journal of Dentistry, 6 (4), 445-453 (2012).

8. Lim, W. H. et al. Downregulation of Wnt causes root resorption. Am J Orthod Dentofacial Orthop, 146 (3), 337-345 (2014).

9. Seifi, M., Eslami, B. \& Saffar, A. S. The effect of prostaglandin E2 and calcium gluconate on orthodontic tooth movement and root resorption in rats[J]. The European Journal of Orthodontics, 25 (2), 199-204 (2003).

10. Shimizu, M. et al. Interleukin-17/T-helper 17 cells in an atopic dermatitis mouse model aggravate orthodontic root resorption in dental pulp. Eur J Oral Sci, 121 (2), 101-110 (2013).

11. Pardo, A. et al. Role of matrix metalloproteinases in the pathogenesis of idiopathic pulmonary fibrosis. Respir Res, 17, 23 (2016).

12. Bonnans, C., Chou, J. \& Werb, Z. Remodelling the extracellular matrix in development and disease. Nat Rev Mol Cell Biol, 15 (12), 786-801 (2014).

13. Cantarella, G. et al. Levels of matrix metalloproteinases 1 and 2 in human gingival crevicular fluid during initial tooth movement. Am J Orthod Dentofacial Orthop, 2006. 130(5): p. 568 e11-6.

14. Gonzales, C. et al. Repair of root resorption 2 to 16 weeks after the application of continuous forces on maxillary first molars in rats: a 2- and 3-dimensional quantitative evaluation. Am J Orthod Dentofacial Orthop, 137 (4), 477-485 (2010).

15. Krishnan, V. Root Resorption with Orthodontic Mechanics: Pertinent Areas Revisited. Aust Dent J, 62 (Suppl 1), 71-77 (2017).

16. Yamaguchi, M. et al. Low-energy laser irradiation facilitates the velocity of tooth movement and the expressions of matrix metalloproteinase-9, cathepsin $\mathrm{K}$, and alpha(v) beta(3) integrin in rats. Eur $\mathrm{J}$ Orthod, 32 (2), 131-139 (2010).

17. Deryugina, E. I. et al. Matrix metalloproteinase-2 activation modulates glioma cell migration[J]. Journal of Cell Science, 110 (Pt 19), (19):2473-2482 (1997).

18. Ren, Y., Maltha, J. C. \& Kuijpers-Jagtman, A. M. Optimum force magnitude for orthodontic tooth movement: A systematic literature review[J]. Angle Orthod, 73 (1), 86-92 (2003).

19. Brudvik, P. \& Rygh, P. The initial phase of orthodontic root resorption incident to local compression of the periodontal ligament[J]. The European Journal of Orthodontics, 15 (4), 249-263 (1993).

20. Brudvik, P. \& Rygh, P. Non-clast cells start orthodontic root resorption in the periphery of hyalinized zones[J]. The European Journal of Orthodontics, 15 (6), 467-480 (1993). 
21. Brudvik, P. \& Rygh, P. Root resorption beneath the main hyalinized zone[J]. The European Journal of Orthodontics, 16 (4), 249-263 (1994).

22. Brudvik, P. \& Rygh, P. Multi-nucleated cells remove the main hyalinized tissue and start resorption of adjacent root surfaces[J]. Eur J Orthod, 16 (4), 265-273 (1994).

23. Brudvik, P. \& Rygh, P. Transition and determinants of orthodontic root resorption-repair sequence.[J]. Eur J Orthod, 17 (3), 177-188 (1995).

24. Brudvik, P. \& Rygh, P. The repair of orthodontic root resorption: An ultrastructural study[J]. The European Journal of Orthodontics, 17 (3), 189-198 (1995).

25. Visse, R. \& Nagase, H. Matrix metalloproteinases and tissue inhibitors of metalloproteinases: structure, function, and biochemistry. Circ Res, 92 (8), 827-839 (2003).

26. Zee, E. V. D., Everts, V. \& Beertsen, W. Cytokine-induced endogenous procollagenase stored in the extracellular matrix of soft connective tissue results in a burst of collagen breakdown following its activation[J]. 1996, 31(7):483-488.

27. Wu, Y. M., Richards, D. W. \& Rowe, D. J. Production of matrix-degrading enzymes and inhibition of osteoclast-like cell differentiation by fibroblast-like cells from the periodontal ligament of human primary teeth. J Dent Res, 78 (2), 681-689 (1999).

28. Linsuwanont, B. et al. Expression of matrix metalloproteinase-9 mRNA and protein during deciduous tooth resorption in bovine odontoclasts[J]. Bone (New York), 31 (4), 0-478 (2002).

\section{Tables}

Table 1: Comparison of the expression of MMP-2 in the pressure side of different groups $(\bar{x} \pm s$, $n=35)$

\begin{tabular}{|c|c|c|c|}
\hline Group & Pressure side & $\mathrm{F}$ & $\mathrm{P}$ \\
\hline Control & $0.05204 \pm 0.01121$ & & \\
\hline $1 d$ & $0.07202 \pm 0.00772^{(1)}$ & & \\
\hline $3 d$ & $0.13658 \pm 0.03379$ & & \\
\hline $5 d$ & $0.14846 \pm 0.01381^{3}$ & 9.438 & 0.000 \\
\hline $7 d$ & $0.18989 \pm 0.03968^{(9)}$ & & \\
\hline $14 d$ & $0.11641 \pm 0.04738^{(100(9)}$ & & \\
\hline 21d & $0.06993 \pm 0.01133^{(00}$ & & \\
\hline
\end{tabular}

(1) is compared with the control group, $\mathrm{P}>0.05$; (2) is compared with the $1 \mathrm{~d}$ group, $\mathrm{P}>0.05$;

(3) is compared with the $3 \mathrm{~d}$ group, $\mathrm{P}>0.05$; (4) is compared with the $5 \mathrm{~d}$ group, $\mathrm{P}>0.05$; (5) is compared with the $7 \mathrm{~d}$ group, $\mathrm{P}>0.05$; (6) is compared with the $14 \mathrm{~d}$ group, $\mathrm{P}>0.05$. 


\section{Figures}
A.
B.
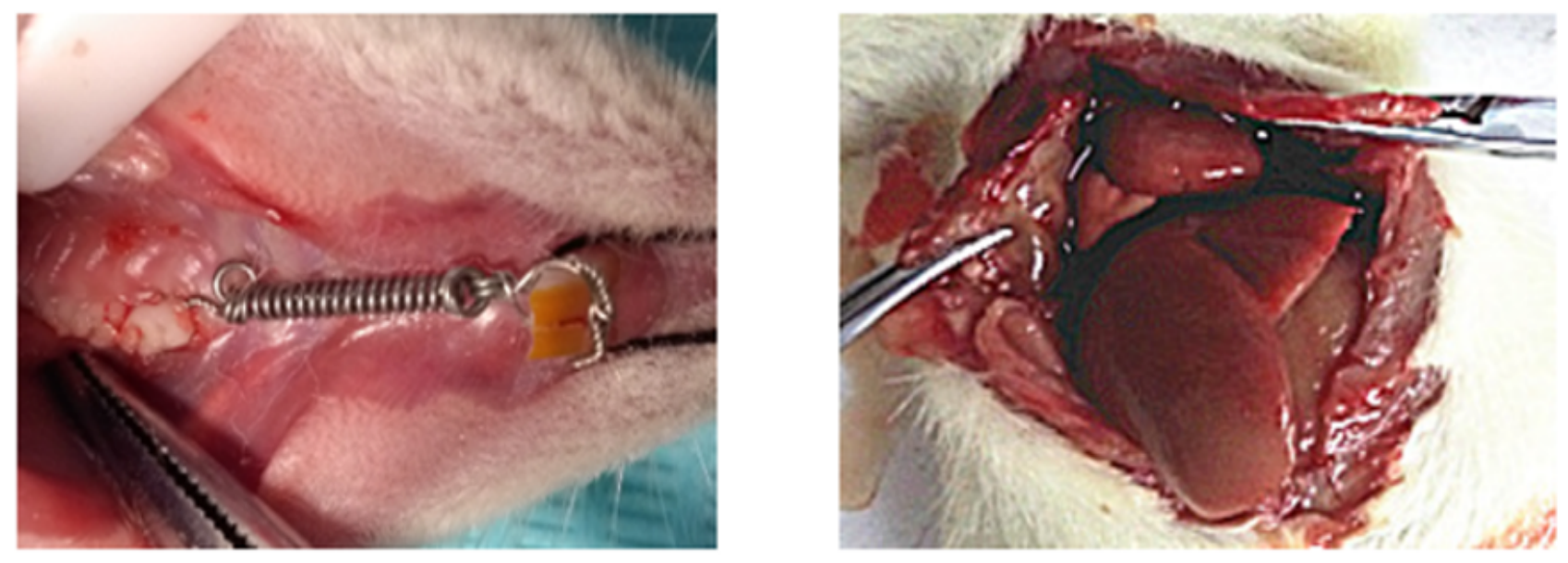

Figure 1

\section{Figure 1}

Experiment Procedure A: Closed nickel-titanium coil spring between the first right molar and incisors. B: Rat heart perfusion 

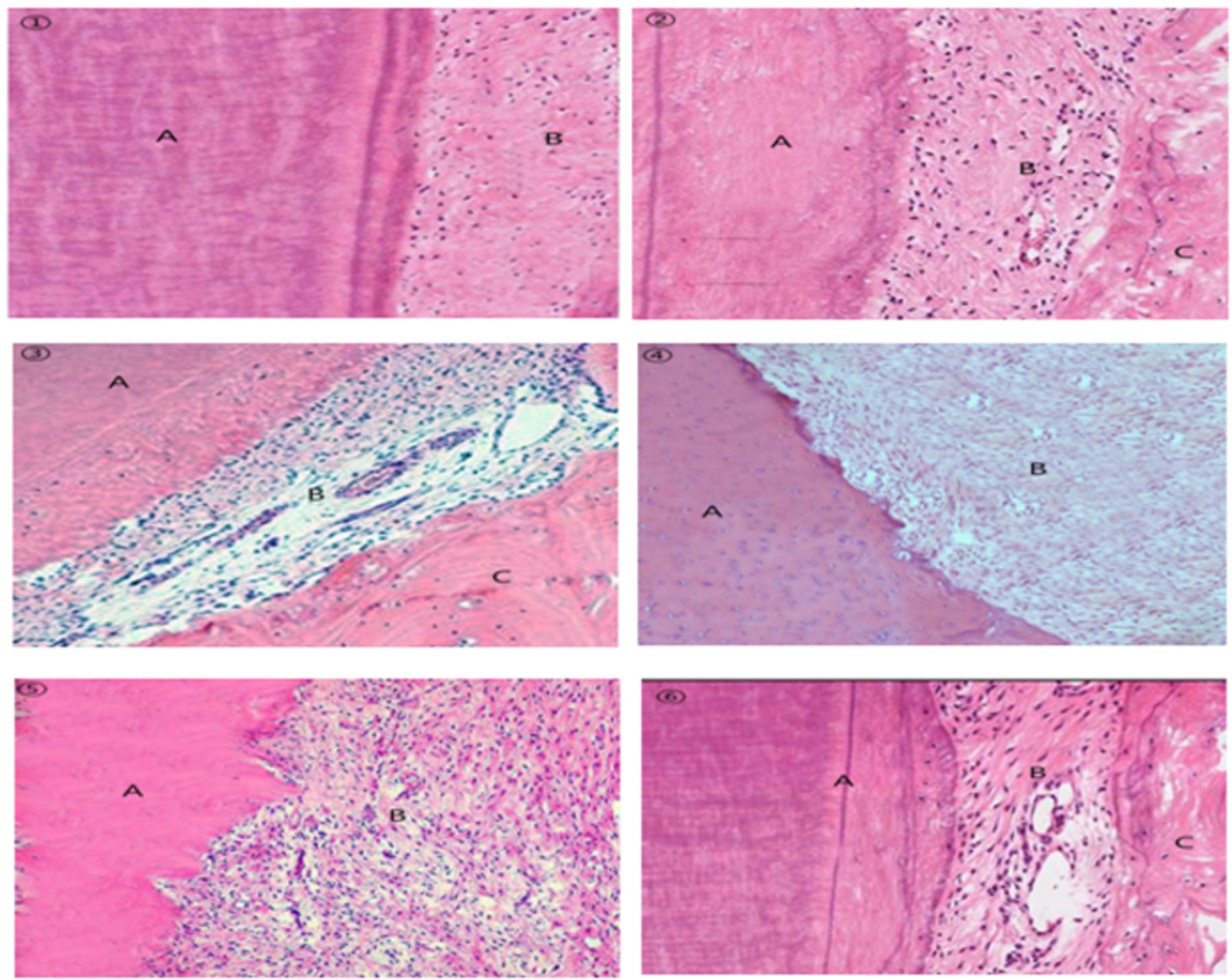

\section{Figure 2}

\section{Figure 2}

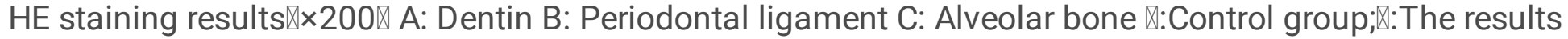

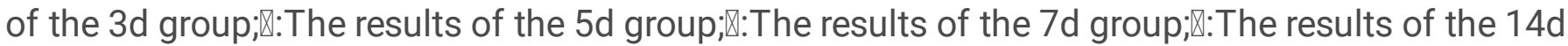
group;: $:$ The results of the $21 \mathrm{~d}$ group 

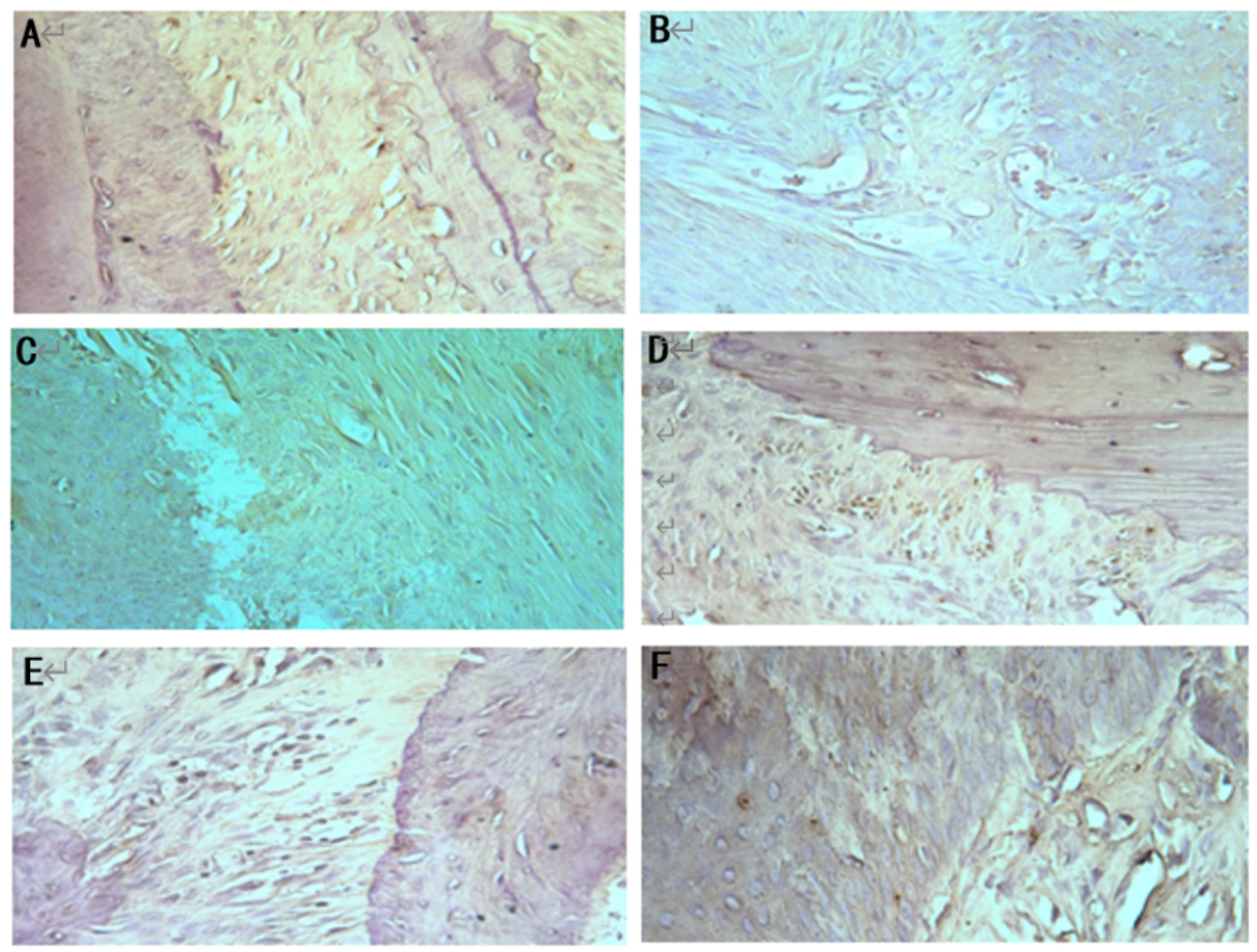

Figure 3

Figure 3

Immunohistochemistry results A: The expression of MMP-2 in the control group (×400) B: The expression of MMP-2 in the $3 d$ group $₫ \times 400 \rrbracket$ C: The expression of MMP-2 in the $5 d$ group $\varangle \times 400 \otimes D$ : The expression of MMP-2 in the $7 \mathrm{~d}$ group $\varangle \times 400 \otimes \mathrm{E}$ : The expression of MMP-2 in the $14 \mathrm{~d}$ group $(\times 400) \mathrm{F}$ : The expression of MMP-2 in the $21 \mathrm{~d}$ group $₫ \times 400 \rrbracket$ 


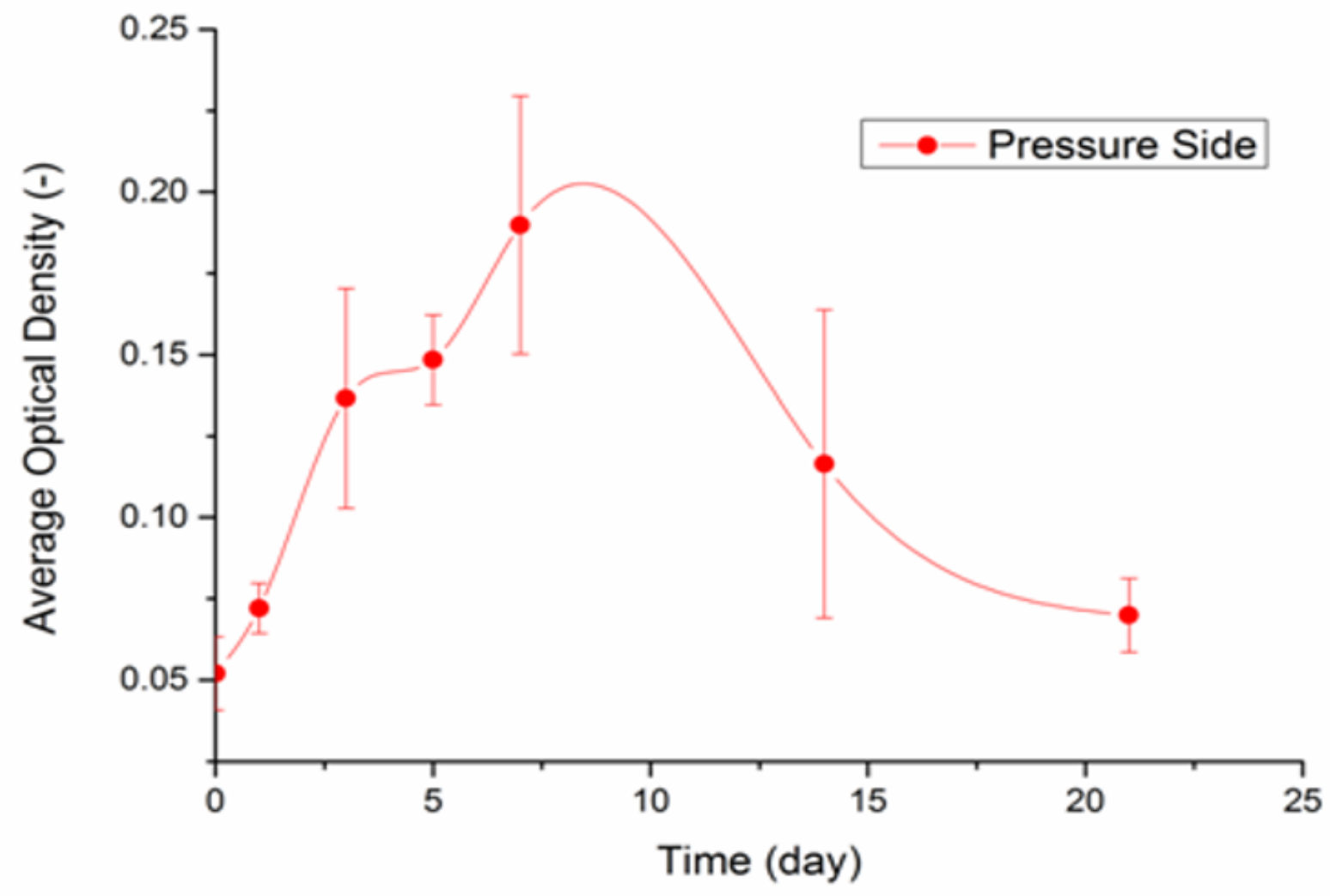

\section{Figure 4}

\section{Figure 4}

The mean optical density of the pressure side of the MMP-2 was expressed over time 